\title{
Presynaptic Recording of Quanta from Midbrain Dopamine Neurons and Modulation of the Quantal Size
}

\author{
Emmanuel N. Pothos, Viviana Davila, and David Sulzer \\ Departments of Neurology and Psychiatry, Columbia University, New York, New York 10032, and Department of \\ Neuroscience, New York State Psychiatric Institute, New York, New York 10032
}

\begin{abstract}
The observation of quantal release from central catecholamine neurons has proven elusive because of the absence of evoked rapid postsynaptic currents. We adapted amperometric methods to observe quantal release directly from axonal varicosities of midbrain dopamine neurons that predominantly contain small synaptic vesicles. Quantal events were elicited by high $\mathrm{K}^{+}$or $\alpha$-latrotoxin, required extracellular $\mathrm{Ca}^{2+}$, and were abolished by reserpine. The events indicated the release of 3000 molecules over $200 \mu \mathrm{sec}$, much smaller and faster events than quanta associated with large dense-core vesicles previously recorded in vertebrate preparations. The number of dopamine molecules per quantum increased as a population to $380 \%$ of
\end{abstract}

Quantal release provides the basic unit of neurotransmission, and analysis of quantal events is essential to understanding synaptic activity. However, quanta at many synapses have not been recorded because of the lack of rapid postsynaptic currents. Prominent examples are the dopamine (DA) synapses of the nigrostriatal and mesocorticolimbic tracts. These projections play important roles in long-term potentiation, working memory, reward, attention and motivation, and motor control. Mesocorticolimbic/nigrostriatal dysfunction underlies Parkinson's disease, schizophrenic psychosis, tardive dyskinesia, drug dependence, and forms of dementia. Despite the fundamental roles for these neurons in brain function and disease, the understanding of their synaptic properties lags behind systems mediated by fast-acting transmitters because of the lack of approaches to observe quantal release and its modulation.

Recently, several techniques have provided the means for presynaptic observation of quantal secretion. Capacitance recordings provide for the observation of vesicle exocytosis, although the technique is not sufficiently sensitive to resolve individual small synaptic vesicles in neurons (Parsons et al., 1994; Rieke and Schwartz, 1994; Mennerick and Matthews, 1996). Evanescentwave fluorescence microscopy provides for the observation of large dense-core granule secretion (Steyer et al., 1997). Destain-

\footnotetext{
Received Jan. 15, 1998; revised March 13, 1998; accepted March 24, 1998.

E.N.P. is an Aaron Diamond Foundation Fellow and the recipient of a 1995 National Alliance for Research on Schizophrenia and Depression Young Investigator Award. This work was supported by a National Institute on Drug Abuse (NIDA) Shannon Award (to D.S.) and by NIDA, the Parkinson's Disease Foundation, National Alliance for Research on Schizophrenia and Depression, and the Aaron Diamond Foundation. We are grateful to Drs. Stephen Rayport, Steven Siegelbaum, and Serge Przedborski for discussion of this manuscript; to Johanna Bogulavsky, Jevons Liu, and Irina Ryjak for technical assistance; and to David Cherkas for characterizing the electrode response.

Correspondence should be addressed to Dr. David Sulzer, Black Building, Room 305, Columbia University, 650 West 168th Street, New York, NY 10032.

Copyright (C) 1998 Society for Neuroscience $\quad 0270-6474 / 98 / 184106-13 \$ 05.00 / 0$
}

controls after glial-derived neurotrophic factor (GDNF) exposure and to $350 \%$ of controls after exposure to the dopamine precursor L-dihydroxyphenylalanine (L-DOPA). These results introduce a means to measure directly the number of transmitter molecules released from small synaptic vesicles of CNS neurons. Moreover, quantal size was not an invariant parameter in CNS neurons but could be modulated by neurotrophic factors and altered neurotransmitter synthesis.

Key words: $\alpha$-latrotoxin; amperometry; dopamine; electrochemistry; exocytosis; GDNF; L-DOPA; midbrain; quantal analysis; quantal release; recycling; synaptic vesicles; VMAT

ing of the endocytic tracer FM1-43 occurs in quantal units under limited stimulation (Ryan et al., 1997). However, these techniques cannot measure the basic parameters of quantal size and duration. On the other hand, postsynaptic receptor saturation or modification may disguise changes in quantal size, and the millisecond-range responses of postsynaptic currents are insufficient to delineate the duration of exocytic release from small synaptic vesicles. Therefore, the number of molecules released and the duration of the quantal events in the CNS have been subjects of conjecture (Almers and Tse, 1990; Van der Kloot, 1991; Garris et al., 1994; Tong and Jahr, 1994).

Recently, amperometric techniques have measured presynaptic monoaminergic quantal events of endocrine cells (Leszczyszyn et al., 1991), invertebrate neurons (Bruns and Jahn, 1995; Chen et al., 1995), and peripheral neurons (Zhou and Misler, 1995). These studies examined neurotransmitter release by large densecore vesicles (150-1000 nm in diameter), with the single exception of transmitter release from axonal stumps of the leech Retzius cell, where both large dense-core and small synaptic vesicles ( $\sim 50 \mathrm{~nm}$ in diameter) were observed (Bruns and Jahn, 1995).

In the present study we have adapted amperometric recording to observe quantal events caused by small synaptic vesicle exocytosis from midbrain DA neurons. These data provide direct measurement of the number of molecules and the duration of release of quantal events in CNS neurons, which prove to be very different from those associated with large dense-core vesicles in peripheral neurosecretory cells. The data also indicate that the number of molecules released per quanta can be modulated presynaptically, i.e., that the size of the CNS release event is not invariant. This would be particularly important for systems in which transmitter overflows from a presynaptic site to multiple neighboring postsynaptic sites, as occurs with midbrain DA terminals (Garris et al., 1994). The approach introduced in this 
paper thus provides a new means for analysis of the fundamental units of CNS transmission.

\section{MATERIALS AND METHODS}

Primary cultures. Postnatally derived cultures of midbrain neurons were prepared as described earlier (Rayport et al., 1992) except that serumfree medium was used (Mena et al., 1997). Recordings were performed 3-6 weeks postplating. We did not observe amperometric spikes in younger cultures, perhaps because of delayed maturation of the synaptic machinery after the dissociation procedure and continued neurite outgrowth during this period (Burke et al., 1998); such delays are not unusual for studies of synaptic function in neuronal cultures. The medium contained the antioxidants selenium and catalase and did not contain ascorbic acid. The major released oxidizable species detected by HPLC with electrochemical detection (HPLC-EC) were identified as DA and its metabolites dihydroxyphenylacetic acid (DOPAC) and homovanillic acid. The cultures did not contain other monoaminergic cell types (Rayport et al., 1992). For glial-derived neurotrophic factor (GDNF)-treated cultures, GDNF (10 ng/ml; Intergen, Purchase, NY) was added to the normal growth medium on the day of plating.

Experimental conditions. Normal recording medium contained (in $\mathrm{mM}$ ) $150 \mathrm{NaCl}, 2 \mathrm{KCl}, 10 \mathrm{HEPES}, 1 \mathrm{MgCl}_{2}, 25$ glucose, and $1.2 \mathrm{CaCl}_{2}, \mathrm{pH}$ 7.4. $\mathrm{Ca}^{2+}$-free medium eliminated $\mathrm{CaCl}_{2}$ and included $1.2 \mathrm{~mm}$ EGTA. Stimulation media used were $20-80 \mathrm{~mm} \mathrm{~K}^{+}$iso-osmotically substituted for $\mathrm{Na}^{+}$in the normal medium, or $\alpha$-latrotoxin $(\alpha$-LTX; $20 \mathrm{nM})$ provided by Dr. Alexander Petrenko (New York University). Secretagogues were applied from a distance of $20-40 \mu \mathrm{m}$ from the recording electrode by using gentle (3-6 psi) air pressure (Picospritzer, General Valve, Fairfield, NJ) for 2-6 sec for a total application of $\sim 7-20 \mu$ l. Therefore, final secretagogue levels at the neuron were more dilute. Because quantal size was not different for the different secretagogues (see Fig. 2B), $\alpha$-LTX sometimes was prepared in high $\mathrm{K}^{+}$medium. Recordings took place at room temperature $\left(25^{\circ} \mathrm{C}\right)$, and experiments were completed within 90 min of removal from the incubator. For the data reported in Table 2 and Figure 6, L-dihydroxyphenylalanine (L-DOPA) exposures were for $30 \mathrm{~min}$ at $36.7^{\circ} \mathrm{C}$, and the L-DOPA-containing medium was replaced with fresh physiological saline before recording.

Visualization of recycling compartments. We measured FM1-43 fluorescence in 23 neurite regions of interest (ROI; $1-3 \mu \mathrm{m}^{2}$ area) during seven trials before and after local microinjection of $40 \mathrm{~mm} \mathrm{~K}^{+}$. A $40 \times$ Plan-Neofluar 1.3 numerical aperture oil immersion lens and standard fluorescein filter sets (Zeiss, Oberkochen, Germany) were used. Digital images were obtained with a Photometrics SenSys PVCAM 12-bit camera (Tucson, AZ) using IPLab Spectrum software (Signal Analytics, Vienna, VA) for acquisition and National Institutes of Health Image 1.60 software for quantification. Two images were acquired in $\mathrm{Ca}^{2+}$-free media (with $1.2 \mathrm{~mm}$ EGTA) 5 min apart. Two subsequent images were taken, the first after a single extracellular exposure to $40 \mathrm{mM} \mathrm{K}^{+}$for 10 sec (local perfusion as above) and the other after three additional $10 \mathrm{sec}$ exposures to $40 \mathrm{~mm} \mathrm{~K}^{+}$. The fluorescence arbitrary units are the mean emission per pixel per ROI as indicated by the digital camera, with a maximum emission of 4096 units.

Electrochemical recording. Carbon fiber electrodes were manufactured as described (Pothos et al., 1996), and potential was applied via an Axopatch 200B amplifier (Axon Instruments, Foster City, CA). Electrodes with background $>0.9 \mathrm{pA}$ root mean square (rms; four-pole $5 \mathrm{kHz}$ Butterworth filter) or unstable cyclic voltammograms when tested in a solution of $10 \mu \mathrm{M}$ DA were rejected.

The response speed of the system was characterized by applying a 5 $\mathrm{mV}$ square pulse through the carbon fiber. The pulse rise time was 5 $\mu \mathrm{sec}$, the maximum sampling rate of the boards we used (ITC-18, Instrutech, Great Neck, NY). The resulting current showed exponential growth and decay, as expected from the relationship $I=C d V / d t$. For 5 - $\mu \mathrm{m}$-diameter electrodes, the time constant of the response was $143 \pm$ $9 \mathrm{kHz}(n=4$ electrodes). The rapid response of the carbon fiber electrode indicates that there was essentially no time distortion for events of duration in excess of $20 \mu \mathrm{sec}$, depending on the filter. However, a 100 $\mathrm{kHz}$ four-pole Bessel filter provided unacceptable background levels. We selected a sampling rate of $20 \mu \mathrm{sec}$ (to provide $>9$ points per event) with a $10 \mathrm{kHz}$ four-pole Bessel filter. Background noise levels in the data are attributable mostly to inherent properties of the electrode. The digital noise of the system is below resolution; the 16 bit digital-to-analog converter across the range we recorded $( \pm 250 \mathrm{pA})$ resolves points $\sim 7.5$ fA apart, at least two orders of magnitude less than the typical rms electrode noise.
Electrodes were lowered gently onto the varicosities until a gentle deformation of the surrounding membrane was observed. Baseline current was recorded for $6-48 \mathrm{sec}$ before the application of the secretagogue. If quantal events were noted during the first $30 \mathrm{sec}$ after stimulation, recording was continued for $3 \mathrm{~min}$ because of the delayed response observed in protocols that used $\alpha$-LTX. All analysis was performed without further filtering beyond the $10 \mathrm{kHz}$ four-pole Bessel used in acquisition, and spikes appeared as in Figure 6. For the purposes of illustration only, Figures 2 ( $A$ and top trace of $B$ only) and 5 were low-pass-filtered at $1 \mathrm{kHz}$ (Figs. $2 B$ and 5 were filtered digitally by GW Instruments, Somerville, MA; Fig. $2 A$ was filtered by a four-pole Bessel filter) to improve signal/background at low temporal resolution. The bottom traces of Figure $2 B$ were filtered digitally at $10 \mathrm{kHz}$ to provide a sharp cutoff at that frequency and to eliminate a few extremely rapid (1 data point) current transients in intervals between events.

Amperometric spikes were identified as events with $\geq 3$ data points (i.e., a $40 \mu \mathrm{sec}$ interval) $>4.5$ times the rms noise of the baseline. This cutoff excluded transients observed during background recordings in the bath. The cutoff amplitude was typically $\sim 7 \mathrm{pA}$ above baseline (rms noise with a four-pole $10 \mathrm{kHz}$ Bessel filter was typically $\sim 1.4 \mathrm{pA}$ ). The event width was the duration between (1) the baseline intercept of the maximal incline from the baseline to first point that exceeded the cutoff and (2) the first data point after the maximal amplitude that registered a value less than or equal to baseline. The maximum amplitude $\left(i_{\max }\right)$ of the event was the highest value within the event. The width at half height $\left(t_{1} / 2\right)$ was determined by the duration of the spike trace at one-half of the maximal amplitude. To resolve quantal size, we determined the total charge of the event between the baseline intercepts and the number of molecules estimated by the relation $n=Q / n F$, where $Q$ is the charge, $n$ is the number of electrons donated per molecule, and $F$ is Faraday's constant $(96,485$ coulombs per equivalent). Quantal sizes reported in this study were based on an assumption of two electrons donated per oxidized molecule of DA (Ciolkowski et al., 1994), although monoamines donate four electrons under conditions of restricted diffusion (Ciolkowski et al., 1994; Bruns and Jahn, 1995); the lower estimate was derived in a study of DA oxidation in an analogous system (Ciolkowski et al., 1994).

HPLC with electrochemical detection. Monoamines and metabolites were measured by HPLC-EC. For measurement of DA and metabolites in whole cultures, sister cultures $(n=4-5$ per condition) were exposed first to high $\mathrm{K}^{+}$(40 mM, $2 \mathrm{~min}$ ), and the extracellular medium was stored at $-80^{\circ} \mathrm{C}$ for injection. Then the cells were scraped and extracted with $0.1 \mathrm{~N}$ perchloric acid for the measurement of the remaining intracellular levels. Separation was obtained by a Velosep $10 \mathrm{~cm}$ RP-18 $3 \mu \mathrm{m}$ column (Applied Biosystems, Foster City, CA). Detection was performed by a coulometric detector (Coulochem II, ESA, Bedford, MA) with a guard cell set at $-40 \mathrm{mV}$ and an analytical cell at $400 \mathrm{mV}$. The mobile phase contained $6.9 \mathrm{gm} / 1$ sodium phosphate, $80 \mathrm{mg} / 1$ EDTA, $750 \mathrm{mg} / 1$ heptane sulfonic acid, and 4.5\% methanol, pH 3.6 (phosphoric acid).

Immunostaining. Tyrosine hydroxylase (TH) immunostaining was performed as described (Przedborski et al., 1996). Rabbit anti-vesicular monoamine transporter (VMAT2) primary polyclonal antibody was obtained from Dr. Robert Edwards (University of California, San Francisco). After fixation (4\% paraformaldehyde for $30 \mathrm{~min}$ ), cultures were incubated for $48 \mathrm{hr}$ with an anti-VMAT2 antibody diluted at 1:100 and visualized with biotinylated goat anti-rabbit antibody and diaminobenzidine/horseradish peroxidase reaction (Vectastain Elite kit, Vector Laboratories, Burlingame, CA). Digital images were acquired with a CCD camera (Photometrics). The measurement of VMAT-labeled varicosity dimensions and distributions was performed with National Institutes of Health Image software.

Electron microscopy. For electron microscopic observations, the preparation was as reported (Sulzer and Rayport, 1990) except that the ice-cold fixative (1.5\% glutaraldehyde in $0.1 \mathrm{M}$ phosphate buffer) was exchanged rapidly with the culture medium over $5 \mathrm{sec}$. This provided far better preservation of 5-hydroxydopamine (5-OHDA) label than slower room temperature fixation in the presence of $400 \mu \mathrm{M} \mathrm{CaCl} \mathrm{Cl}_{2}$, which provided good membrane preservation (Sulzer and Rayport, 1990; Rayport et al., 1992).

Statistics. Comparison of populations used the Kolmogorov-Smirnov statistic (GBStat, Dynamic Microsystems, Silver Spring, MD), a standard nonparametric procedure routinely used for the analysis of quantal populations detected by postsynaptic recording (Van der Kloot, 1991). ANOVA with repeated measures was used to compare changes in fluorescence emission. 


\section{RESULTS}

\section{Amperometric recording from axonal varicosities}

To observe directly the quantal release from a presynaptic site of a central neuron, we examined axonal varicosities of midbrain DA neurons derived from rat neonates and cultured for 3-6 weeks. DA neurons identified by immunoreactivity to $\mathrm{TH}$ antibodies (Fig. $1 A_{1}$ ) exhibited neurites that commonly were observed to extend over $1 \mathrm{~mm}$ in length. The axons displayed presynaptic varicosities that were $0.5-4 \mu \mathrm{m}$ in length (mean $=1.7 \mu \mathrm{m}$; Table 1 ), as indicated by immunostaining for VMAT2, and often were in apparent contact with cell bodies (Fig. $1 A_{2}$; Table 1).

Such sites showed exocytosis of recycling structures, as observed with the stimulation-dependent destaining of the fluorescent endocytic tracer FM1-43 (Fig. 1B). The mean fluorescence was reduced (Fig. 1C) from $1004 \pm 129$ [mean \pm SEM, mean fluorescence arbitrary units per region of interest (ROI)] in the first baseline image (cultures maintained in $\mathrm{Ca}^{2+}$-free medium) and $967 \pm 100$ units in the second baseline image $\left(\mathrm{Ca}^{2+}\right.$-free $)$ to $743 \pm 88$ units after one exposure to $40 \mathrm{~mm} \mathrm{~K}^{+}(10 \mathrm{sec})$ and $562 \pm 105$ units after three additional exposures to $40 \mathrm{mM} \mathrm{K}^{+}(10$ sec each). The observed reduction was significant, as shown by one-way ANOVA with repeated measures $\left(n=23 \mathrm{ROI} ; F_{(3,91)}=\right.$ $12.9868 ; p<0.0001)$. Post hoc Student's $t$ test pairwise comparisons showed that the fluorescence reduction was significant only in the images after $40 \mathrm{~mm} \mathrm{~K}^{+}$exposure $(t=3.53$ and 4.82 with the first image after $40 \mathrm{mM} \mathrm{K} \mathrm{K}^{+}$paired to each of the two baselines, respectively, $p<0.01 ; t=5.30$ and 4.87 for the subsequent post- $40 \mathrm{~mm} \mathrm{~K}{ }^{+}$image, $\left.p<0.01\right)$. Furthermore, there was a significant reduction in fluorescence when the first post-40 $\mathrm{mM} \mathrm{K}^{+}$images were compared with the subsequent images $(t=$ 3.77; $p<0.01$ ). No significant difference was found between the two baseline images per site, which suggests that the observed destaining after stimulation is attributable to exocytosis and not to bleaching of the fluorescent signal.

We used two techniques that assisted in the identification of sites for recording: (1) intracellular labeling to illuminate candidate axonal varicosities and (2) the selection of areas where multiple processes clearly overlap, because these showed a higher incidence of VMAT2 stain (see below). The detection of exocytic release of DA was performed by applying $+700 \mathrm{mV}$ to a $5-\mu \mathrm{m}-$ diameter carbon fiber electrode placed directly on the exposed upper surface $(\sim 20 \%$ of surface area) of such varicosities. When we used these techniques, $\sim 5-10 \%$ of the sites that were examined showed amperometric events. The low fraction of responding areas was not attributable to a low representation of DA neurons, which range from $\sim 20-40 \%$ of the neuronal population and display extensive axonal branching. We suspect that many DAergic axonal varicosities were not electrochemically active because the releasing site was not oriented directly to the electrode surface or was occluded by other neurites or glial sheaths.

All of the sites that showed amperometric events and subsequently were fixed and processed for immunocytochemistry were found to be TH-immunolabeled $(n=12)$. In instances of apparent release at cell bodies, subsequent TH or VMAT2 immunostaining indicated that DA varicosities were under the electrode in close contact with the cell bodies.

In some cases, neurites were labeled with Lucifer yellow $(0.3 \%)$ that was injected during whole-cell patch clamp to visualize varicosities (Fig. $2 A)$. Depolarization by high $\mathrm{K}^{+}(20-80 \mathrm{~mm})$ evoked generally low numbers of amperometric events $(2.6 \pm 1.2$ events, mean \pm SEM; $n=13$ sites where events were observed)
(Fig. $2 A_{3}$, top trace). Because DA diffuses rapidly and the events were mostly $<500 \mu \mathrm{sec}$ in duration, only events directly under the electrode face were likely to have produced distinct amperometric spikes (Schroeder et al., 1992). An elevated baseline was observed sometimes, presumably because of diffusional broadening of DA release from nearby fusion events not directly under the electrode. Amperometric events were dependent on extracellular $\mathrm{Ca}^{2+}$ (Fig. $2 A_{3}$, middle and bottom traces). No events were elicited by the application of physiological saline, and event detection was abolished reversibly when the voltage applied to the electrode was decreased to $0 \mathrm{mV}$. Although we found Lucifer yellow label to be useful for identifying varicosities associated with given cells, the protocol was inefficient, and we generally selected sites that displayed several overlapping neurites.

Amperometric events also were elicited by application of $\alpha$-LTX (Fig. 2B), a secretagogue that induces massive synaptic vesicle exocytosis and sustained transmitter release (Petrenko, 1993). High $\mathrm{K}^{+}$or $\alpha$-LTX elicited amperometric events of identical size (Fig. 2B) although apparent overlapping events were observed with $\alpha$-LTX (see below), whereas such events were rare $\left(<1 \%\right.$ of events) with high $\mathrm{K}^{+}$stimulation. The average number of release events per stimulation was $4 \pm 2$ ( $n=19$ sites). However, in subsequent observations there were rare instances of massive release with $>200$ quanta after a single $\alpha$-LTX application. After subsequent immunostaining for VMAT2 or TH, we could discern no morphological differences between sites that released many events and those that released few.

Amperometric spikes were almost never observed after the application of high $\mathrm{K}^{+}$in $\mathrm{Ca}^{2+}$-free conditions (one event from 50 sites, 4400 molecules) or after exposure to the VMAT inhibitor reserpine (no events from 23 sites after $1 \mu \mathrm{M}$ reserpine for 90 min), a treatment that depletes intracellular and releasable DA stores by $97 \%$ in this system (Sulzer et al., 1996). However, in the case of $\alpha$-LTX, release events could be observed in the absence of extracellular $\mathrm{Ca}^{2+}(n=2$ sites in 16 trials), as has been reported for other neuronal preparations after exposure to this secretagogue (Zhou and Misler, 1995).

In 80 sites where amperometric spikes were observed, only three spontaneous events in control cultures $(3700 \pm 700$ molecules, mean \pm SEM) were recorded during a $\geq 6$ sec period before secretagogue application, consistent with the tendency of midbrain DA neurons to not produce spontaneous action potentials in vitro (Rayport et al., 1992). Hippocampal neurons were used as a control preparation because they do not release detectable monoamines and are not $\mathrm{TH}$-immunopositive $\left(\mathrm{TH}^{+}\right.$; data not shown); no current spikes were detected from hippocampal processes after high $\mathrm{K}^{+}$or $\alpha$-LTX stimulation $(n=35$ sites $)$.

There were a few very large events $(48,900 \pm 12,000$ molecules; $n=5$ ) in the control group that were $>10$-fold greater than the $\mathrm{SD}$ of the rest of the population distribution. These large events were evoked by $\alpha$-LTX $(n=4)$ and high $\mathrm{K}^{+}(n=1)$. Analogous infrequent large postsynaptic potentials, termed "giant" miniature end plate potentials, have long been noted at the neuromuscular junction (Van der Kloot, 1991).

To examine the vesicle population capable of accumulating DA in our system, we identified DA synaptic vesicles under electron microscopy by uptake of the DA analog 5-OHDA, using a rapid glutaraldehyde fixation technique that we found to be very effective at fixing vesicular 5-OHDA. This analog produces an electron-dense reaction product after exposure to osmium tetroxide. We found that a majority $(>99 \%)$ of vesicles in the DA varicosities in this system are small synaptic vesicles with diame- 

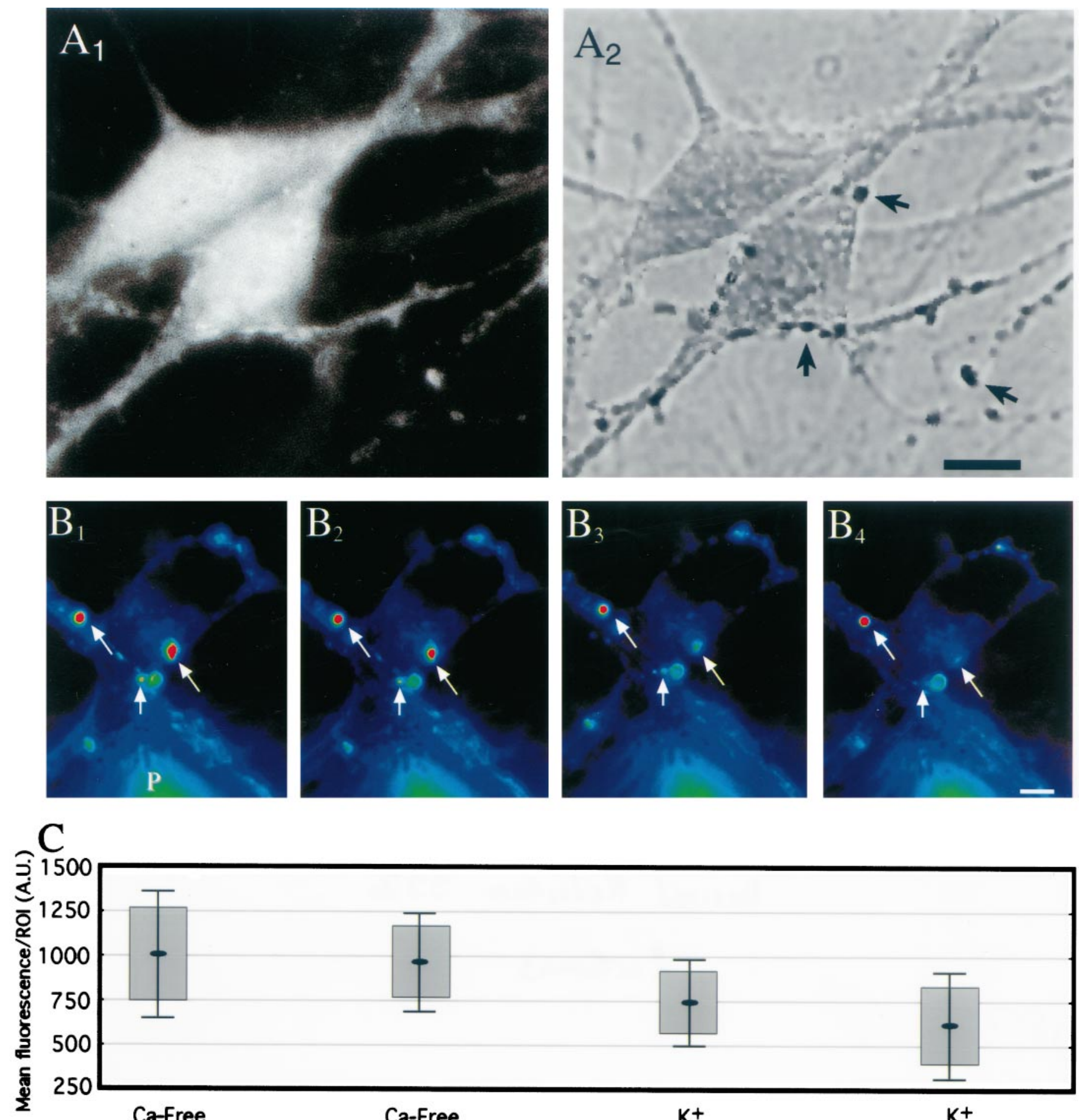

\section{Ca-free}

Ca-Free

$\mathrm{K}^{+}$

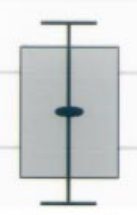

$\mathrm{K}^{+}$

Figure 1. $A_{l}$, Ventral midbrain dopamine (DA) neurons in culture were immunostained for tyrosine hydroxylase (TH), using a fluorescein-conjugated secondary antibody. $A_{2}$, The same cell pair immunostained for the brain vesicular monoamine transporter (VMAT2) and visualized by a horseradish peroxidase/diaminobenzidine reaction product. The VMAT2-labeled varicosities (examples indicated by arrows) measured $0.5-4 \mu \mathrm{m}$ in diameter at their longest axis and occurred both with and without obvious contact to other neurons. Scale bar, $5 \mu \mathrm{m}$. $B_{1}, B_{2}$, Presumed presynaptic compartments (examples indicated at arrows) were labeled by the endocytic tracer FM1-43 (4 $\mu \mathrm{M}$ in the presence of $6 \mu \mathrm{M}$ nicotine for 1 min; the nicotinic acetylcholine receptor is presynaptic at midbrain DA neurons) (Marshall et al., 1997). The neurons were maintained in $\mathrm{Ca}^{2+}$-free medium for 10 min with little decrement in label. The figures display the label at 5 and $10 \mathrm{~min}$. The perfusion pipette $(P)$ extends from the base and appears green in the pseudocolored images because of the autofluorescence. $B_{3}, B_{4}$, Then these sites were stimulated with $40 \mathrm{~mm} \mathrm{~K}^{+}$medium $(10$ sec), decreasing the label at several sites in the path of the perfusion. Three further applications of $40 \mathrm{~mm} \mathrm{~K}{ }^{+}$medium $(10 \mathrm{sec})$ induced further decrement of FM1-43 label, demonstrating that the sites studied contain organelles that recycle on stimulation. The site indicated by the top left arrow, which is farther from the perfusion pipette, shows less decrement than sites closer to the pipette. Scale bar, $5 \mu \mathrm{m}$. C, Quantitation of decrease in mean fluorescence per region of interest (ROI) after stimulation $(n=23$ sites $)$. Each box and whiskers symbol corresponds to the exposures as indicated in the figure above. The mean is indicated by the black horizontal cross on the vertical bars, the limits (whiskers) at the ends of the bars indicate the maximum and minimum values for the data set, and the box limits indicate the 5th and 95th percentiles of the data set. 
Table 1. Effects of one-time GDNF exposure on DA release, distribution of VMAT2-stained varicosities, and varicosity morphology

\begin{tabular}{|c|c|c|c|c|c|c|c|}
\hline & DA release & $\begin{array}{l}\text { Percentage of } \mathrm{TH}^{+} \\
\text {cell bodies }\end{array}$ & $\begin{array}{l}\mathrm{VMAT}^{+} \text {intersite } \\
\text { distance }\end{array}$ & $\mathrm{VMAT}^{+} /$area & $\begin{array}{l}\mathrm{VMAT}^{+} \\
\text {surface area }\end{array}$ & $\mathrm{VMAT}^{+}$length & $\mathrm{VMAT}^{+}$width \\
\hline Control & $0.54 \pm 0.14$ & $18.11 \pm 0.0$ & $24.7 \pm 0.15$ & $1.69 \pm 0.13$ & $0.66 \pm 0.04$ & $1.70 \pm 0.04$ & $0.37 \pm 0.01$ \\
\hline GDNF & $1.52 \pm 0.19$ & $37.90 \pm 1.4$ & $22.9 \pm 0.11$ & $1.69 \pm 0.13$ & $0.46 \pm 0.03$ & $1.42 \pm 0.04$ & $0.31 \pm 0.01$ \\
\hline
\end{tabular}

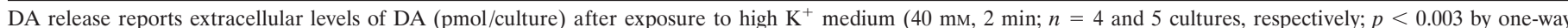

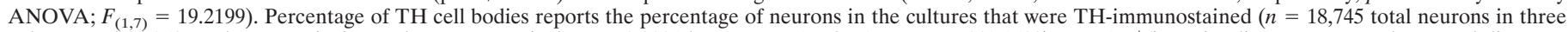

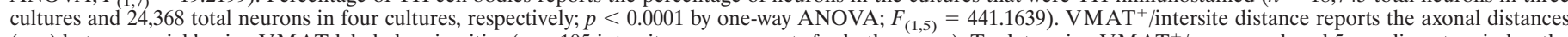

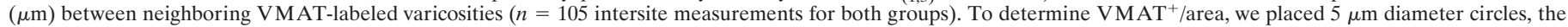

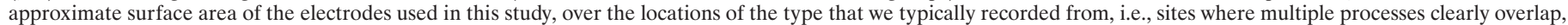

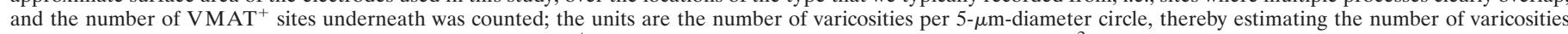

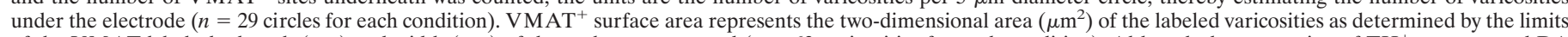

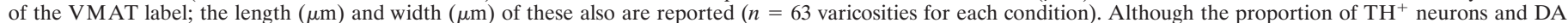

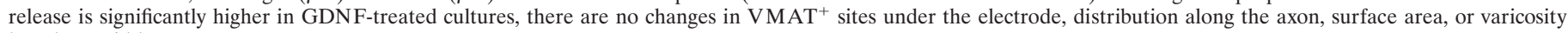
length or width.

ters of 40-60 nm (Fig. 3), with very few large dense-core vesicles. [Additional electron micrographs of axonal varicosities in ventral midbrain cultures using a conventional fixation technique that provides improved plasma membrane preservation but relatively poor preservation of intravesicular 5-OHDA are found in previous publications of Rayport et al. (1992) and Sulzer and Rayport (1990); these figures also show uptake of the analog into small vesicles and a far less frequent ( $\leq 1$ per terminal/section) presence of large dense-core vesicles.] The relative presence of large densecore vesicles in terminal cross sections is over-represented because of stereological considerations, because a large dense-core vesicle will appear in more thin sections than a small synaptic vesicle. Therefore, both in vivo (Nirenberg et al., 1997) and these cultures, small synaptic vesicles in DA terminals appear to be the major organelle responsible for the uptake and release of DA.

Postsynaptic recordings at neuromuscular junction and central preparations demonstrate that the number of quanta released is a function of extracellular $\mathrm{Ca}^{2+}$ level (Van der Kloot, 1991). Using $40 \mathrm{~mm} \mathrm{~K}^{+}$as a secretagogue, we elicited only one spike in $\mathrm{Ca}^{2+}$-free medium, which was identical to the mean quantal size at normal $\mathrm{Ca}^{2+}$ levels $\left(1.2 \mathrm{~mm}\right.$; see above). When $\mathrm{Ca}^{2+}$ was elevated to $6 \mathrm{~mm}$, which would be expected to increase the probability of simultaneous exocytosis, the quantal sizes were also not different from those at $1.2 \mathrm{~mm}(2700 \pm 300$ molecules; $n=11$ events, 4 sites). The finding that quantal sizes were not altered by changes in extracellular $\mathrm{Ca}^{2+}$ suggests that most of the events that were recorded were monoquantal.

\section{Effects of GDNF}

Classically, the postsynaptic response to the secretion of neurotransmitter released because of a single synaptic vesicle fusion event is termed quantal size in quantal analysis and hence can be modulated by either presynaptic or postsynaptic mechanisms. However, modulation of quantal size in CNS neurons has not been demonstrated. One approach to determine whether presynaptic quantal size may be modulated is to examine neurotrophic factors, which are known to facilitate transmitter release as well as to promote neuronal survival (for review, see Kang and Schuman, 1995; Thoenen, 1995; Stoop and Poo, 1996).

After the initial characterization of quantal release, we examined this issue under conditions designed to mimic effects reported in vivo. In the rodent a single injection of GDNF, which activates the receptor tyrosine kinase Ret (Durbec et al., 1996; Jing et al., 1996), is neurotrophic for midbrain DA neurons (Hebert et al., 1996) and results in a delayed (1-3 week) elevation of $\mathrm{TH}$ expression and the potentiation of stimulation-dependent
DA release (Beck et al., 1996). Therefore, to examine the effects of GDNF on presynaptic modulation, we cultured ventral midbrain neurons for 3-6 weeks with or without $10 \mathrm{ng} / \mathrm{ml} \mathrm{GDNF}$ in the extracellular medium at the time of plating; the factor was not replaced.

GDNF-exposed cultures displayed a twofold increase in the presence of $\mathrm{TH}^{+}$-labeled cell bodies, similar to studies conducted with embryonically derived cultures (Lin et al., 1993) and postnatally derived cultures (Burke et al., 1998) (Fig. 4A,B, Table 1). To determine the effect of GDNF exposure on overall depolarization-evoked DA release, we used HPLC-EC. GDNF increased the DA release evoked by high $\mathrm{K}^{+}$to $280 \%$ of control levels (Table 1) and the corresponding intracellular DA levels to $400 \%$ (from $6.10 \pm 0.79$ to $24.54 \pm 3.43$ pmol per culture), as measured by HPLC-EC.

To determine whether GDNF increased the density of DA synaptic varicosities, we immunostained for VMAT2 (Fig. 4C,D, Table 1). The number of varicosities that might contribute to amperometric detection was estimated by two methods. First, the number of varicosities per axonal length and the intervaricosity distance along the axon were measured and were found not to change with GDNF exposure (Table 1). However, if more axonal branching occurred, more varicosities might be found under the electrode. Therefore, we counted the number of VMAT2immunostained varicosities present within 5- $\mu \mathrm{m}$-diameter circles at sites with the morphology from which we typically record, i.e., where neurites cross; this was found to be identical under both conditions (Table 1). Finally, the two-dimensional morphology of the varicosities (maximum length along the process axis, maximum width tangential to the process, and area) was not changed by GDNF exposure (Table 1). Therefore, whereas GDNF increased the total number of surviving DAergic neurons and overall axonal outgrowth, it did not alter significantly the morphology or distribution of VMAT2-expressing varicosities along the axons.

We used amperometric recordings from varicosities of GDNFexposed neurons and control cultures to determine the basis of increased DA release. GDNF-treated neurons had a mean quantal size $380 \%$ greater than controls (Table $2 ; p<0.0001$ ). The combination of a higher presence of DA neurons and greater quantal size would suggest that GDNF exposure elevates total DA release per culture to even greater levels than that measured by HPLC. The relatively lower levels of potentiation measured when extracellular levels are examined by HPLC-EC are likely attributable to differences in experimental conditions. In particular, total extracellular DA would underestimate overall quantal 
release, because stimulation of the culture is measured after 2 min of depolarization so that autoreceptors likely have been activated, and there has been significant DA reuptake by the plasma membrane uptake transporter.

\section{Effects of L-DOPA}

TH catalyzes the rate-limiting step for catecholamine synthesis in neurons. If cytosolic levels of DA are rate-limiting for synaptic vesicle uptake, quantal size should be increased by the TH product L-DOPA, which is converted to DA by the cytosolic enzyme aromatic acid decarboxylase. We exposed ventral midbrain DA neurons to $100 \mu \mathrm{M} \mathrm{L}-D O P A\left(30 \mathrm{~min}\right.$ at $25^{\circ} \mathrm{C}$; Fig. 5) or $20 \mu \mathrm{M}$ L-DOPA (30 min at $36.7^{\circ} \mathrm{C}$; Fig. 6, Table 2), which rapidly increases intracellular DA levels in this system as measured by HPLC-EC (HPLC-EC identifies the principal compound released by high $\mathrm{K}^{+}$after L-DOPA as DA; Pothos et al., 1996). In the present study we found that preincubation with L-DOPA elevated quantal size (Table 2), demonstrating that vesicular accumulation is controlled in part by the pool of cytosolic transmitter.

\section{Population distribution of the quantal size}

The protocol used to analyze presynaptic modulation of quantal size could compare either single sites observed before and after a given treatment (e.g., Figs. $2 B, 5$ ) or multiple sites exposed to a single stimulation (e.g., analysis in Table 2 and Fig. 6). Although the examination of single sites would eliminate synapse-tosynapse variation, the low number of events typically induced by high $\mathrm{K}^{+}$makes statistical analysis difficult. Although $\alpha$-LTX induced greater release, and occasionally elicited large numbers of events, it also produces massive $\mathrm{Ca}^{2+}$ currents that may strongly influence subsequent responses, including excitotoxicity. Moreover, catecholamine release is very susceptible to influence from previous stimulations, and chromaffin cells show potentiated quantal release after single high $\mathrm{K}^{+}$exposures (Finnegan et al., 1996). Therefore, although observations at single sites indicated that quantal size was modulated, we suggest that the more stringent analysis in this case was to examine quantal size in groups of sites exposed to the secretagogue once only and to bin the events. Because the effects on quantal size were large, this approach demonstrated highly significant differences between groups.

Individual examples of typical monoquantal events recorded after the first stimulation at neurite varicosities are displayed (Fig. $6 A-C)$. Occasional prespike features that may be analogous to the "foot signals" reported for large dense-core vesicle exocytosis may be present. The total population distributions of the quantal events detected in control, GDNF, and L-DOPA conditions are indicated (Fig. 6D). The quantal size, amplitude, and width are each highly significantly increased in the GDNF and L-DOPA groups in comparison to controls ( $p<0.0001$ in each case; see Table 2).

The distribution of the GDNF and L-DOPA quantal sizes was shifted to the right, and the data indicate a wider variance of values. The untransformed quantal size distribution of the populations displayed a prominent right-hand skew (Fig. 6D) so that the population modes were smaller than the population means (Table 2). The leftmost edge of the control quantal size population was close to the detection limits of the system, $\sim 1000$ molecules (Fig. $6 D$ ), which in turn was attributable to the noise inherent in carbon fiber electrodes. Therefore, there may have been significant very small events not represented in the control group, including vesicles that had not undergone complete refilling during recycling (Fon et al., 1997; Song et al., 1997). However, if the modes of the L-DOPA or
GDNF populations were below detection limits, there would have been a steep rise on the left at the same point (the detection roll-off) as seen in controls. Because this was not the case, we conclude that the majority of exocytic events in these categories was detected. The increased mean values were not attributable to the recruitment of a new subset of vesicles, such as the infrequent large events mentioned above, which would result in a bimodal population; indeed, all events in the GDNF and L-DOPA-treated populations were $<3 \mathrm{SD}$ from the mean.

Quantal sizes recorded from a variety of neuroendocrine cells and the mature neuromuscular junction likewise have been shown not to fit a normal distribution but to have a right-hand skew (Van der Kloot, 1991; Finnegan et al., 1996). However, the cubed roots of the quantal sizes are distributed normally; an explanation for this is that the quantal size is a function of vesicle volume. Because spherical volumes are proportional to $\mathrm{r}^{3}$ and vesicle radii are normally distributed, quantal size should be proportional to the $\mathrm{r}^{3}$ (Bekkers et al., 1990). We found that cubed root transformations of the quantal size population of the present data more closely approached a normal distribution (Fig. 6D).

\section{DISCUSSION}

In this study, quanta from midbrain DA neurons exhibited a unimodal size population that was modulated by exposure to neurotrophic factors and altered transmitter synthesis. The quanta were of far more rapid duration and represented fewer molecules than those from neuroendocrine cells and peripheral neurons, which release $3 \times 10^{4}-10^{6}$ molecules over a period of several milliseconds. The events we recorded were attributable to exocytosis from small synaptic vesicles because (1) small synaptic vesicles are abundant in this preparation, whereas large densecore vesicles are rare, (2) the small synaptic vesicles accumulated a DA analog and so would be competent to provide exocytic release, and (3) in the striatum in vivo, DA axonal varicosities nearly exclusively display small synaptic vesicles, and VMAT2 expression is limited to small synaptic vesicles (Nirenberg et al., 1997); therefore, small synaptic vesicles are the likely sites for the storage and release of DA. Significantly, the leech Retzius cell contains high numbers of both small synaptic vesicles and large dense-core vesicles and exhibits two subpopulations of amperometric events (Bruns and Jahn, 1995). One set exhibits the milliseconds-long durations seen with large dense-core vesicle preparations, whereas a group of rapid events exhibits durations similar to those we report in midbrain DA neurons. Regulation of the quantal size and the duration of the events were not examined in the leech preparation.

We conclude that amperometric events in midbrain DA varicosities were attributable to monoquantal release because (1) randomly coupled events would be expected to be very rare, because only one to three varicosities were in contact with the electrode and the number of release events recorded was low; (2) the patterns of amperometric release events elicited by $\alpha$-LTX or high $\mathrm{K}^{+}$were different, but the events were not of different size; (3) the few spontaneous events recorded were the same size as events elicited with secretagogues; (4) quantal size was not different at $0,1.2$, and $6 \mathrm{mM} \mathrm{Ca}^{2+}$; and (5) the quantal size ${ }^{1 / 3}$ of the GDNF and L-DOPA groups approached a unimodal normally distributed population. Nonetheless, apparent overlapping quanta occasionally were recorded at some sites with $\alpha$-LTX, and a small number of more closely overlapping nonrandom events seem likely to have occurred. To appear as single events, they would have to be highly temporally coordinated, i.e., within 100 

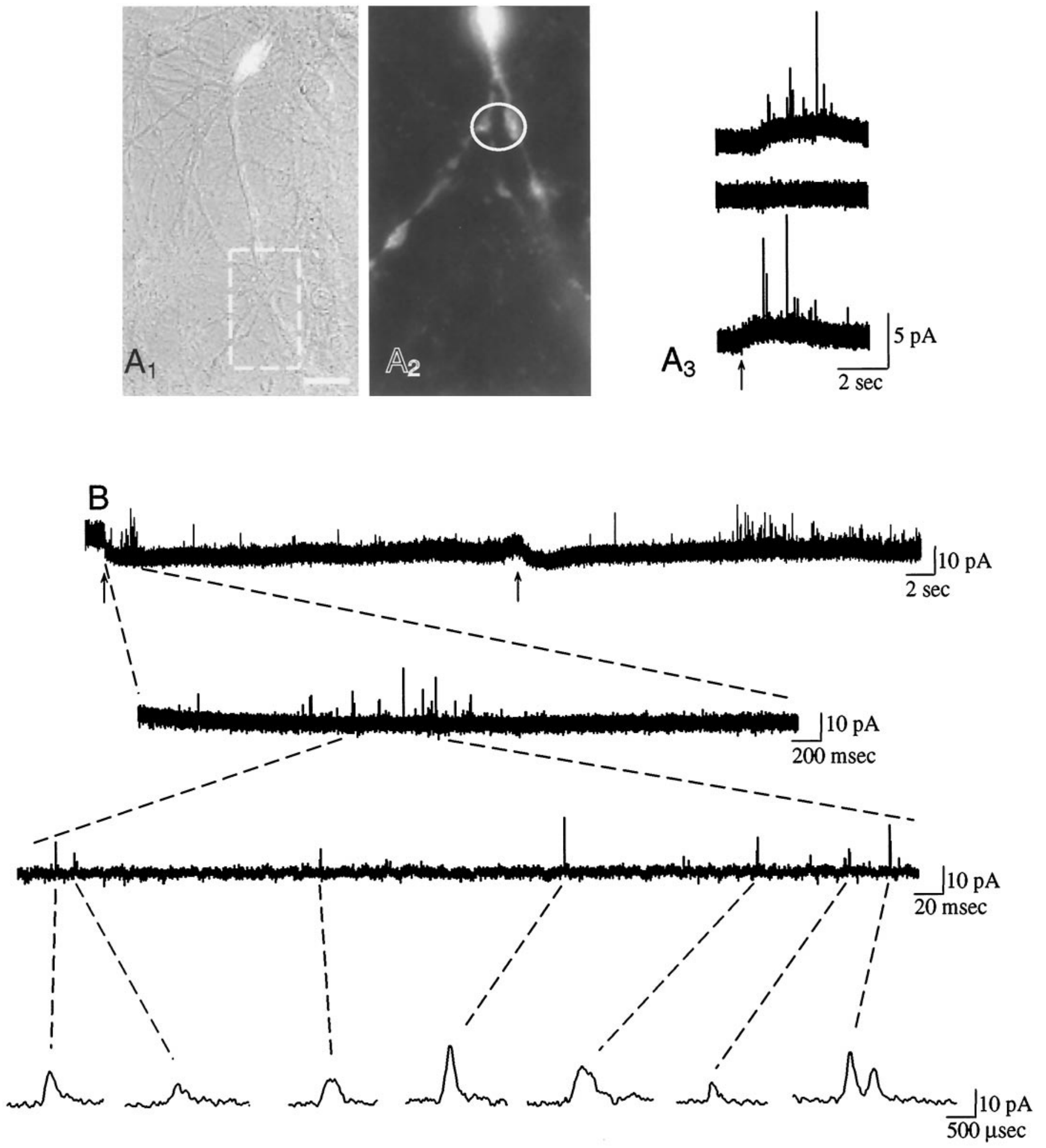

Figure 2. $A_{1}$, A combined fluorescent/incandescent image of a neuron labeled with Lucifer yellow by whole-cell patch clamp. Magnification bar, $10 \mu \mathrm{m}$. $A_{2}$, Fluorescent image of a detail corresponding to the area indicated by the broken box in $A_{1}$. The placement of the $5 \mu \mathrm{m}$ (vertical axis) electrode is indicated by the oval. Two labeled varicosities are apparent under the electrode surface. $A_{3}$, The top trace shows amperometric events at the site in $A_{2}$ after $3 \mathrm{sec}$ of high $\mathrm{K}^{+}$in the presence of $1.2 \mathrm{mM} \mathrm{Ca}^{2+}$ (the arrow indicates the application of high $\mathrm{K}^{+}$for each trace). The events were not evoked by high $\mathrm{K}^{+}$ in $\mathrm{Ca}^{2+}$-free medium (middle trace). Recovery occurred when high $\mathrm{K}^{+}$in the presence of $1.2 \mathrm{~mm} \mathrm{Ca}^{2+}$ was reapplied (bottom trace). Of 14 neurons labeled by Lucifer yellow injection, five showed amperometric spikes from apparent varicosities. For each of three sites so examined, the removal of $\mathrm{Ca}^{2+}$ resulted in the complete cessation of spikes, and spikes resumed after the readministration of high $\mathrm{K}^{+}$with $\mathrm{Ca}^{2+}$. All sites where quanta were observed had $\mathrm{TH}^{+}$processes underneath the electrode, although only three of the five dye-filled neurons in which amperometric events were evoked were themselves $\mathrm{TH}^{+}$; therefore, nondye-filled dopaminergic processes were sometimes under the electrode. $B$, Exemplar amperometric recording contrasting two secretagogues. This culture was exposed to both GDNF and L-DOPA (50 $\mu \mathrm{M}$ for $30 \mathrm{~min}$ ) to promote elevated levels (Figure legend continues) 


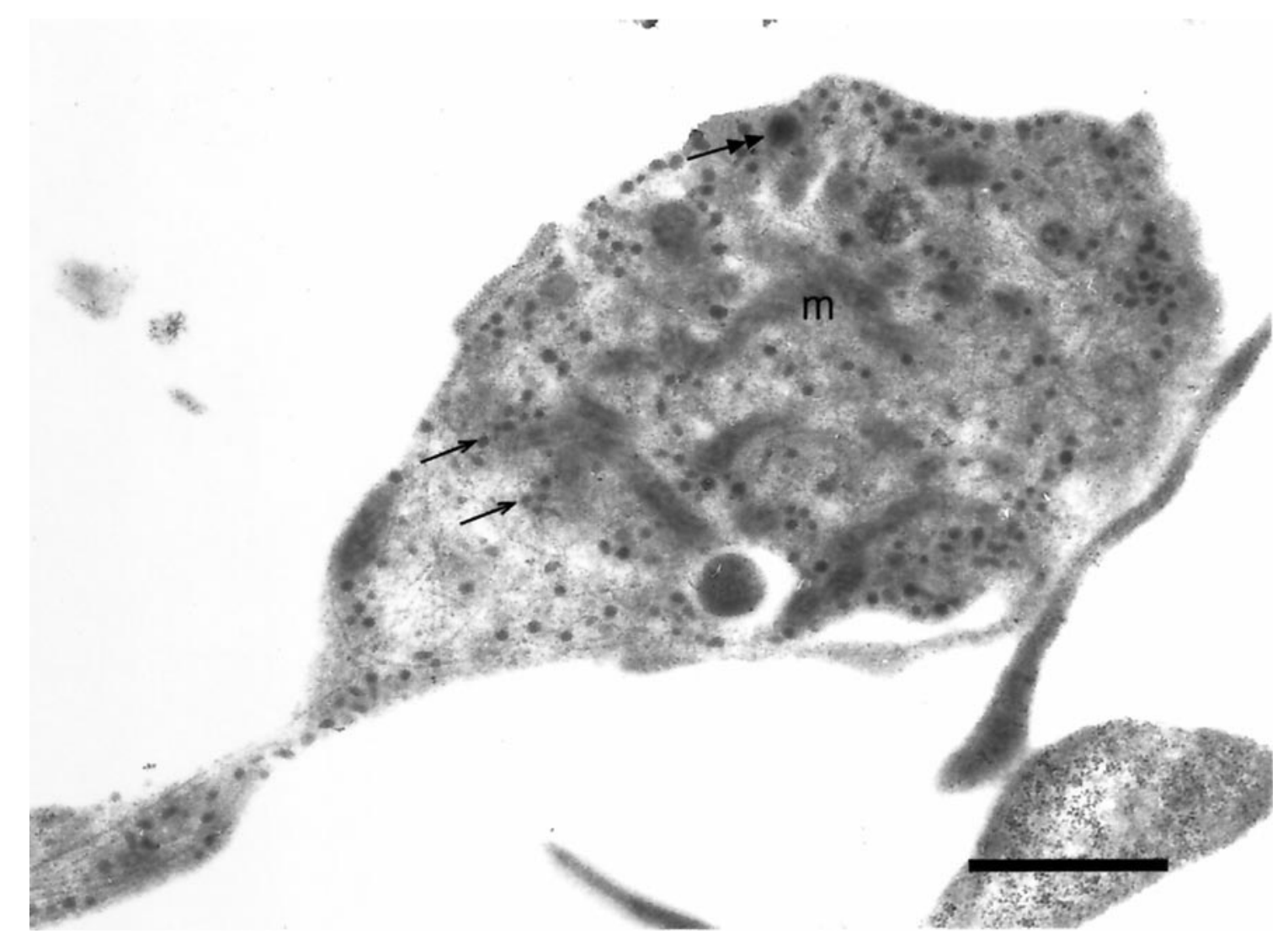

Figure 3. Electron micrograph of a rapidly fixed midbrain DA axonal varicosity in postnatal culture. The preparation was exposed to the osmophilic DA analog 5-hydroxydopamine (5-OHDA; $50 \mu \mathrm{M}$ for $12 \mathrm{hr}$ at $36.7^{\circ} \mathrm{C}$ ) and then fixed with a rapid exposure to glutaraldehyde, which is very effective at preserving intravesicular 5-OHDA. Two hundred and forty-six small synaptic vesicles (40-60 nm in diameter) labeled with 5-OHDA are present (two small vesicles are indicated by single arrows). One large dense-core vesicle is present (160 nm in diameter; double arrow); $m$ indicates a mitochondrion. Ribosomes in a neighboring dendrite are visible at the scale bar. The large dense object in the varicosity could be an endosome, although the quality of the membrane preservation precludes certain identification. Additional micrographs of 5-OHDA-labeled vesicles in midbrain DA culture with the use of conventional fixation methods have been published (Sulzer and Rayport, 1990; Rayport et al., 1992). Scale bar, $1 \mu \mathrm{m}$.

$\mu$ sec. Therefore, the ability to distinguish between "simultaneous" events is at least an order of magnitude better than with previous quantal recording techniques that measure postsynaptic responses of millisecond durations.

An advantage of this approach is that it is adapted for the study of catecholamine release, which is not measured by postsynaptic recording. Quantal release from DA presynaptic varicosities was observed with the electrode placed at the upper surface, clearly indicating that the varicosities can provide extrasynaptic overflow. The amperometric events we report are attributable to the release of DA because (1) other catecholamines were not released in this system as shown by HPLC-EC, (2) the events were abolished rapidly by reserpine, a VMAT inhibitor that depletes vesicular DA but not ascorbic acid (Rebec and Pierce, 1994), (3) events were not observed unless sufficient oxidation potential was ap- plied to the electrode, (4) all sites that provided amperometric events and were processed for immunocytochemistry were $\mathrm{TH}^{+}$, (5) quantal size was increased by exposure to L-DOPA, which promotes synthesis of cytosolic DA, and (6) hippocampal neurons did not produce such events. DA metabolites such as dihydroxyphenylacetic acid (DOPAC) were unlikely to contribute to the signal because there is no evidence that they are sequestered in synaptic vesicles, they are not released in a $\mathrm{Ca}^{2+}$-dependent manner, and they are not substrates for VMAT2 and hence would not be reserpine-sensitive.

\section{Alteration of quantal size}

A basic presynaptic property by which quantal size could be elevated is by the release of more neurotransmitter molecules. We recently have observed this with large dense-core vesicle exocy-

\section{$\leftarrow$}

of DA release. Medium containing $40 \mathrm{mM} \mathrm{K}^{+}$was applied for $2 \mathrm{sec}$ ( first arrow). Nineteen spikes (amplitudes $>4.5 \times$ rms background) were elicited, of which 13 occurred during the perfusion plus 1 sec. $\alpha$-LTX $(20 \mathrm{nM})$ in normal recording medium was applied for $2 \mathrm{sec}$ (second arrow). Then $51 \mathrm{spikes}$ followed, beginning $6.9 \mathrm{sec}$ from the start of the perfusion. A portion of the trace marked by the dashed lines is shown with increased time resolution in the two lower contiguous traces. The dashed lines extending from the lowest contiguous trace indicate those spikes with amplitudes greater than $4.5 \times$ rms background noise. The lowest trace shows these spikes at further increased time resolution. The rightmost pair of events that are displayed appear to overlap slightly at the base. Interevent intervals were $1000 \pm 492 \mathrm{msec}$ (mean $\pm \mathrm{SEM}$ ) for high $\mathrm{K}^{+}$and $1935 \pm 556 \mathrm{msec}$ for $\alpha$-LTX. The quantal sizes elicited by the two secretagogues were not significantly different ( $p=0.24$; KS- $Z=1.0302$, Kolmogorov-Smirnov test). The top trace in $B$ was digitally filtered at $1 \mathrm{kHz}$ to provide better signal-to-noise resolution at low temporal resolution; the lower traces were digitally filtered at $10 \mathrm{kHz}$. Note that the same spikes are visible with both filters. 

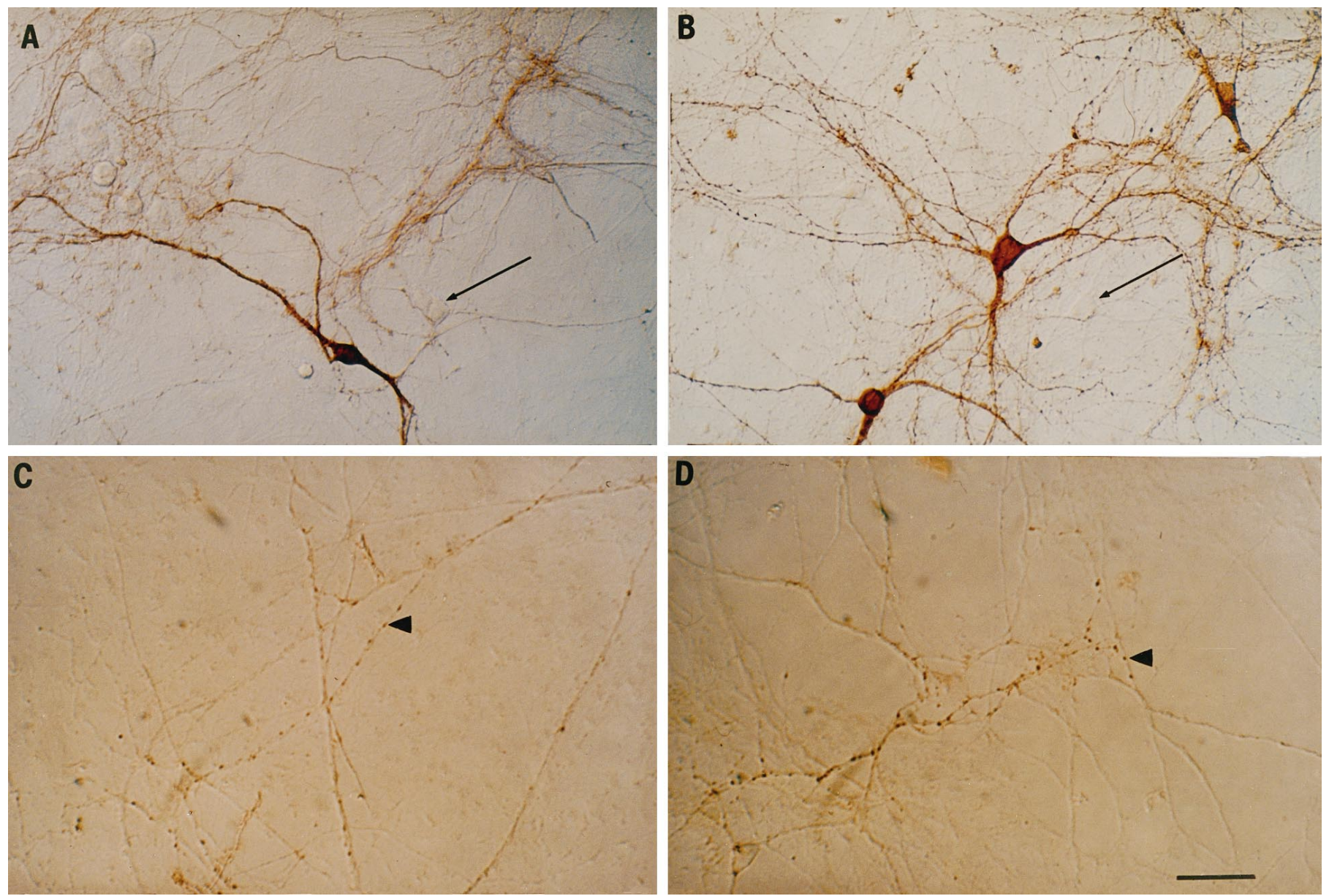

Figure 4. Morphological effect of GDNF on midbrain DA neurons in culture. $A$, Three weeks after plating, TH immunostaining indicates a dense plexus of neurite outgrowth. The arrows indicate TH-unlabeled cell bodies. $B$, Cultures exposed to GDNF display twofold more stained cell bodies (see Table 1) and also maintain a dense plexus of neurites. $C$, VMAT2 immunolabel in a control culture indicates sites containing dopaminergic synaptic vesicles. Arrowheads indicate examples of VMAT-labeled varicosities. D, Shown is a VMAT2 stain of a culture exposed to GDNF. The distribution of VMAT2-labeled varicosities along the axis of the axon and the two-dimensional structure of the varicosities were not altered by GDNF exposure (see Table 1). Scale bar, $50 \mu \mathrm{m}$.

Table 2. Comparison of amperometric events from control neurons and neurons exposed to GDNF or $20 \mu \mathrm{M}$ L-DOPA for $30 \mathrm{~min}$ (mean \pm SEM)

\begin{tabular}{lllll} 
& $\begin{array}{l}\text { Number of } \\
\text { molecules (mean) }\end{array}$ & $\begin{array}{l}\text { Number of } \\
\text { molecules (mode) }\end{array}$ & $i_{\text {max }}(\mathrm{pA})$ & $\begin{array}{l}\text { Width } \\
(\mu \mathrm{sec})\end{array}$ \\
\hline Control & $3000 \pm 300$ & 2300 & $14.6 \pm 1.4$ & $120 \pm 40$ \\
GDNF & $11,400 \pm 700$ & 4300 & $26.8 \pm 1.6$ & $180 \pm 40$ \\
$20 \mu \mathrm{M} \mathrm{L-DOPA}$ & $10,600 \pm 400$ & 5400 & $23.7 \pm 1.0$ & $130 \pm 80$ \\
\hline
\end{tabular}

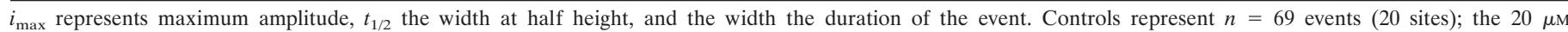

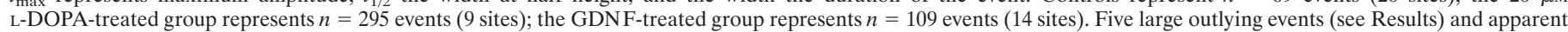

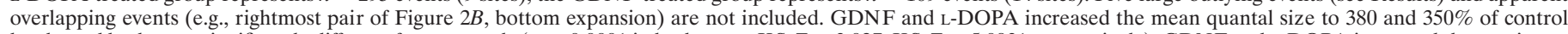

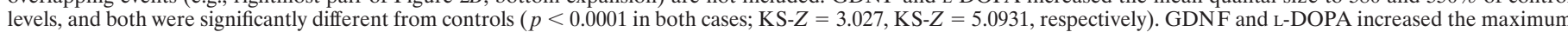

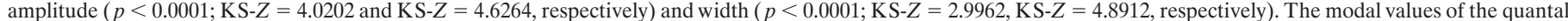

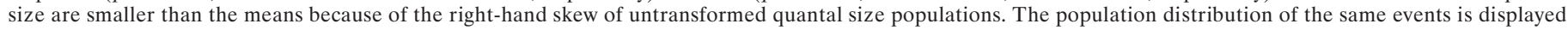
in Figure $6 D$.

tosis in peripheral secretory cell lines by using amperometric recordings of PC12 cells (Sulzer et al., 1995; Pothos et al., 1996), and analogous effects have been reported at the neuromuscular junction (Van der Kloot and Molgo, 1995; Song et al., 1997). In the case of quantal catecholamine release, it is possible that transmitter release from large (150-1000 $\mathrm{nm}$ in diameter) densecore vesicles in PC12 and other neurosecretory cells that contain prominent electron-dense precipitates of transmitter and associated anions differs in fundamental ways from the release caused by exocytosis of small (50 nm in diameter) "clear" synaptic vesicles.

An alternate explanation for elevated quantal size could be that treatments changed the distance of the recording electrode from the release site. This possibility is inconsistent with the following observations. (1) The charge measured with a $\sim 5$ - $\mu$ m-diameter electrode surface remains the same for distances of up to $\sim 1 \mu \mathrm{m}$ (Schroeder et al., 1992). The present experiments used an electrode in physical contact with the plasma membrane so that the 


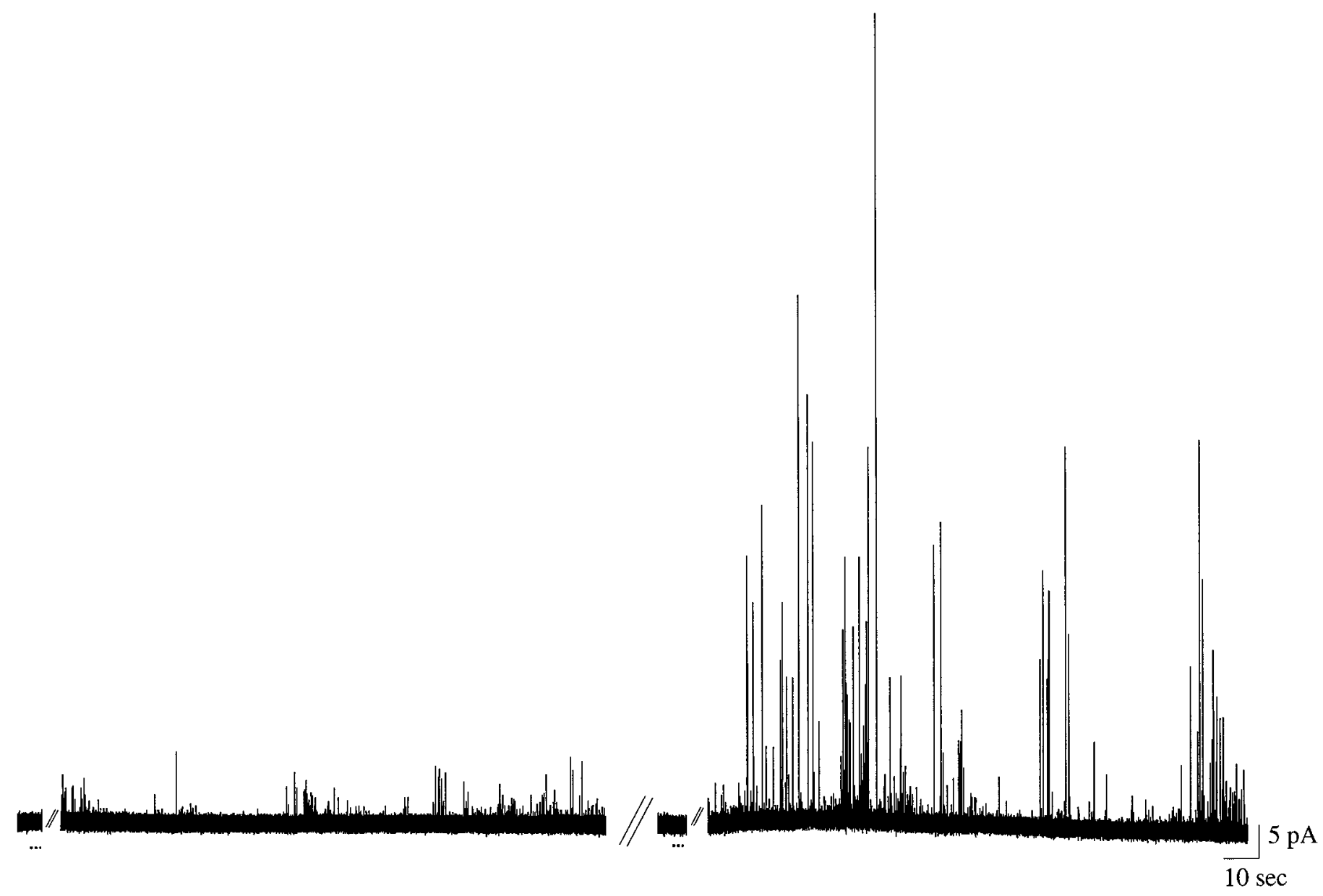

Figure 5. Amperometric recording from a presumed axonal varicosity of a GDNF-exposed neuron before and after exposure to L-DOPA. $\alpha$-LTX (20 $\mathrm{nM}$ ) was perfused for $3 \mathrm{sec}$ ( first dotted line); the trace displays the period of 45-210 sec that follows (events at this site were not observed until 45 sec after $\alpha$-LTX stimulation). The mean quantal size was $10,300 \pm 1000$ molecules $(n=49)$. Then the culture was exposed to $100 \mu \mathrm{M}$ L-DOPA for 30 min, and $\alpha$-LTX was reapplied (second dotted line); the trace displays the period of $45-210 \mathrm{sec}$ that follows. Of the total events elicited ( $n=317$ ), the mean size was 39,700 \pm 3700 molecules; of these, $n=90$ appear to be overlapping events. If apparent overlapping events (e.g., rightmost pair of Fig. $2 B$, bottom expansion) are removed from consideration, the mean quantal size was increased to $18,300 \pm 1600$ molecules $(p<0.0001$ different from control; KS- $Z=$ 2.3389).

electrode is much closer than $1 \mu \mathrm{m}$ to release sites. (2) As the distance between the electrode face and release site increases, the event amplitude decreases, whereas width increases. In contrast, larger quantal sizes result in both an increased amplitude and broader duration (Schroeder et al., 1992; Chow and von Ruden, 1995). Therefore, the results with GDNF and L-DOPA are consistent only with increased quantal size. (3) Control and GDNFtreated DA neurons display VMAT2-labeled axonal varicosities with identical length, width, total area, and intervaricosity distances. Therefore, the surface in contact with the electrode is unchanged. (4) GDNF- and L-DOPA-mediated increases in quantal size were consistent with potentiated total release that was measured independently by HPLC-EC. This technique measures all DA in the culture, distinguishing stimulation-dependent release and intracellular levels. In summary, we suggest that the evidence weighs heavily in support of an increase in quantal size caused by the elevated release of transmitter.

The previous difficulty in demonstrating presynaptic modulation of quantal size in the CNS is attributable in part to the saturation of postsynaptic sites from the contents of a single vesicle (Tang et al., 1994; Edwards, 1995). Nevertheless, regulation of quantal size could play a role in synaptic plasticity for synapses that are either not saturated by a quantum (Bekkers et al., 1990; Tong and Jahr, 1994; Frerking et al., 1995; Liu and Tsien, 1995; Forti et al., 1997) or from which extrasynaptic overflow occurs (Garris et al., 1994; Kullmann et al., 1996). The present results with L-DOPA suggest that the well known control of $\mathrm{TH}$ activity in vivo by second messenger-mediated phosphorylation, neuronal activity, and antipsychotic drugs (Kumer and Vrana, 1996), as well as GDNF (Beck et al., 1996), modulates quantal size. These findings thus indicate a possible physiological role for the regulation of transmitter synthesis.

The results indicate that GDNF can modulate synaptic function even in relatively mature neurons, suggesting a basis for the potential therapeutic use of this factor for Parkinson's disease and other neurodegenerative disorders. In a culture model of synapse formation at the neuromuscular junction, neurotrophins elicit an elevated amplitude of postsynaptic currents (Wang et al., 1995) along with an increased probability of quantal release (Stoop and Poo, 1996). Neurotrophic factors can increase transmitter release via a variety of mechanisms, including potentiation of the number of release events, promotion of additional synapse formation, and elevated quantal size. The present results indicate that elevated quantal size plays a prominent, if not major, role in GDNF- 
A
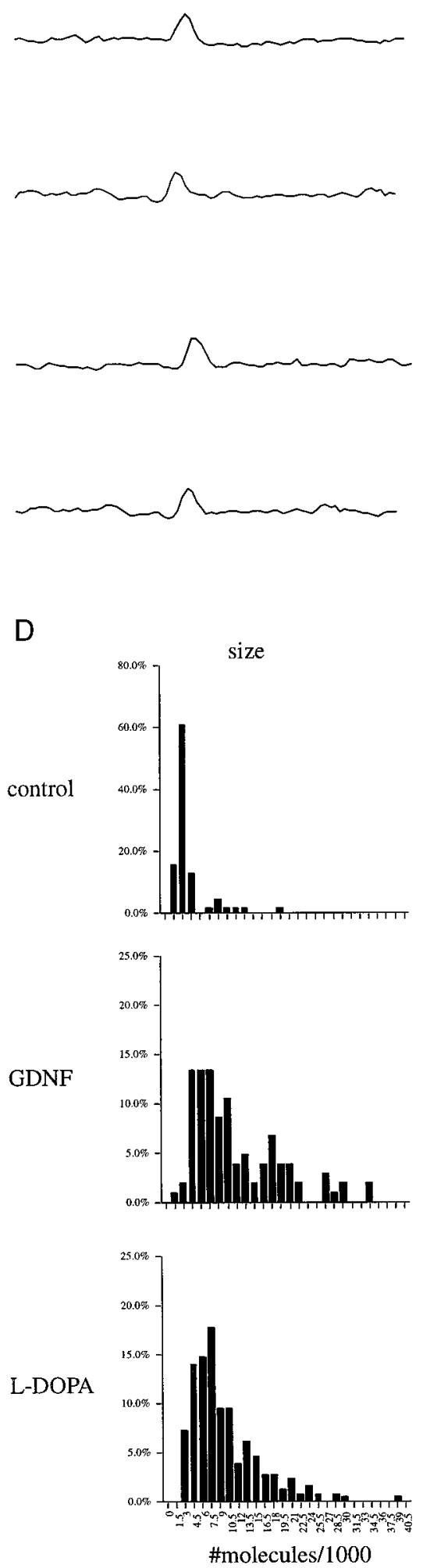

B
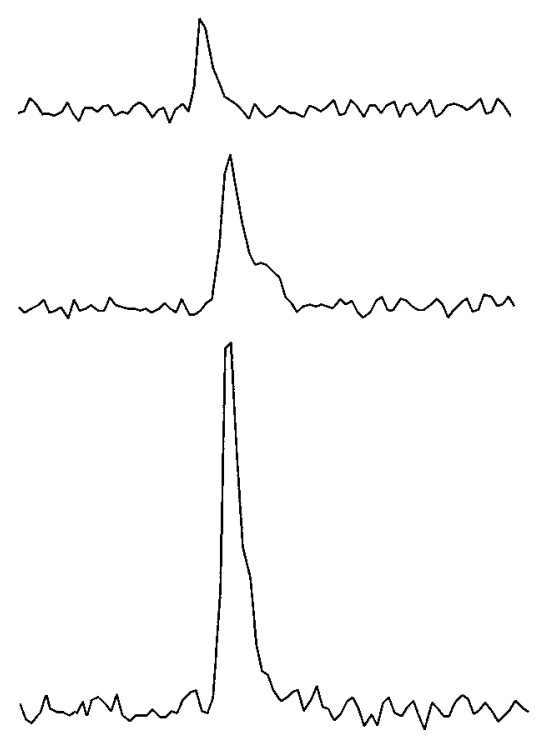

C
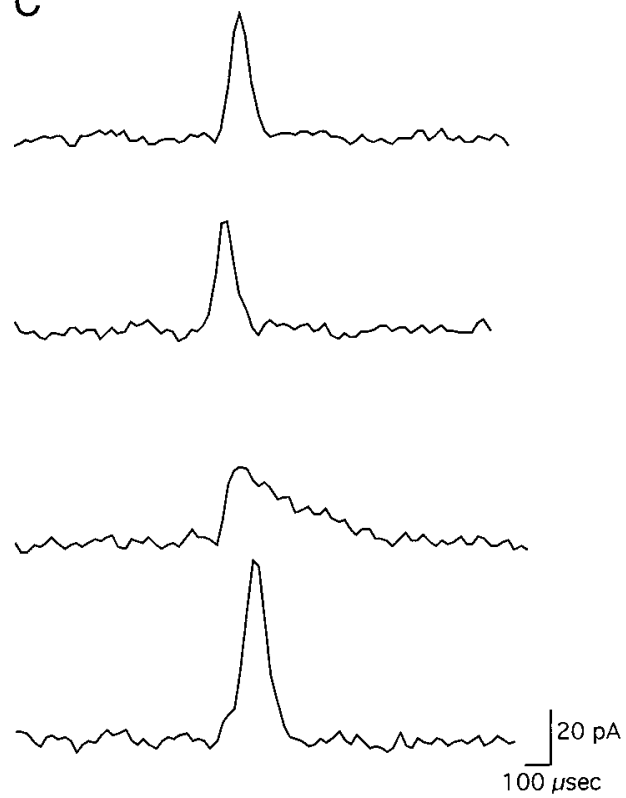
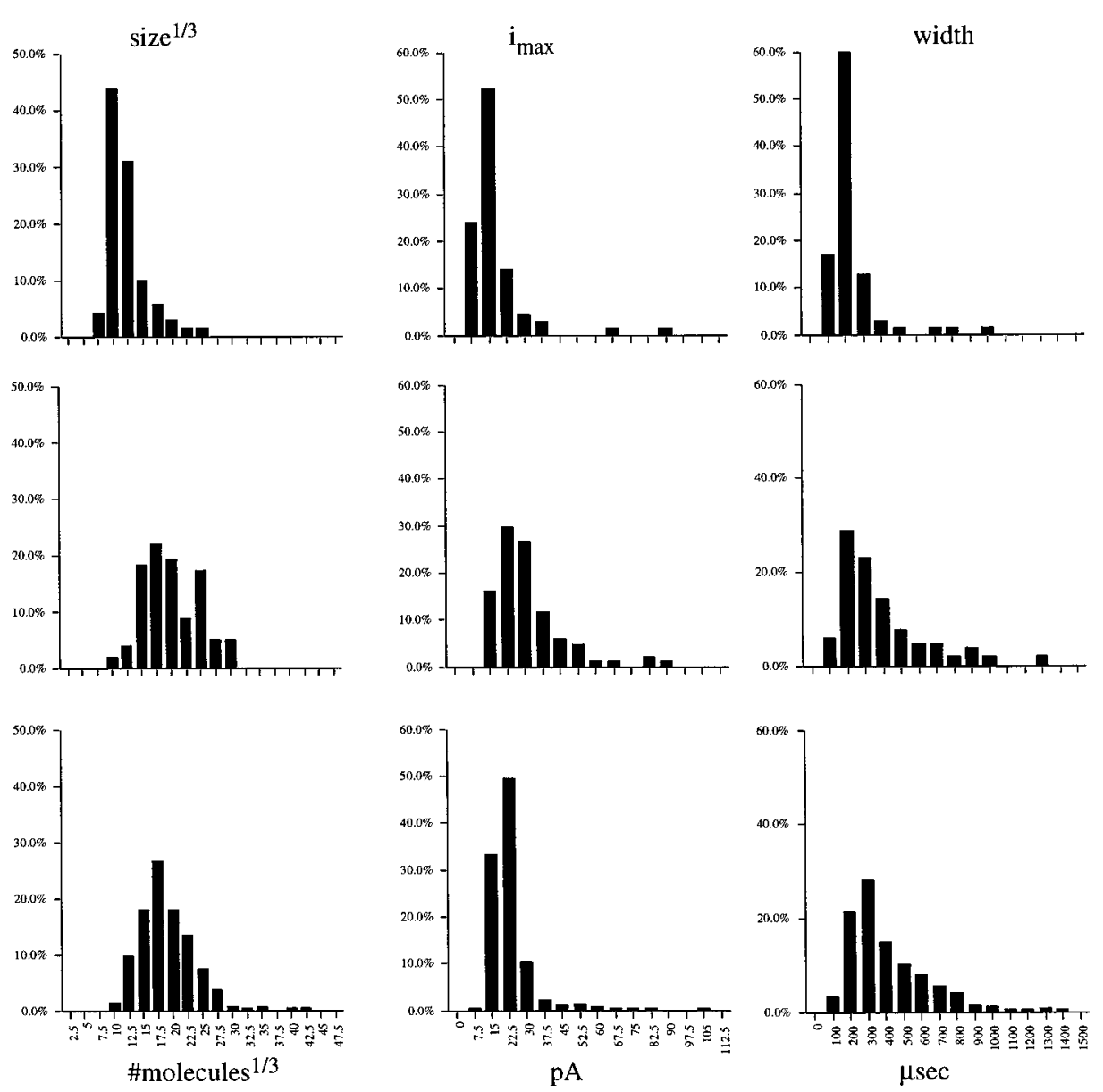

Figure 6. A, Sample amperometric events evoked from control neurons. $B$, Examples of events from GDNF-treated preparations. $C$, Examples of events after exposure to $20 \mu \mathrm{M} \mathrm{L-DOPA}(30 \mathrm{~min})$. D, Distributions of the untransformed (molecules $/ 1000)$ and cubed root transformations (molecules ${ }^{1 / 3}$ ) of quantal sizes, maximum amplitude $(p A)$, and width $(\mu \mathrm{sec})$ of the events reported in Table 2 . In each case, exposure to L-DOPA or GDNF shifted the population parameters to higher values. Note that the control untransformed quantal sizes distribution uses a different $y$-axis scale than the GDNF and L-DOPA groups. 
evoked increased release in this preparation. A variety of mechanisms may underlie this effect by GDNF, such as elevated VMAT per vesicle, potentiation of the vesicle electrochemical gradient, or increased $\mathrm{TH}$ activity.

Although the results do not provide a complete understanding of the action of GDNF beyond demonstrating that more neurotransmitter is synthesized, packaged per synaptic vesicle, and released, the steps underlying the potentiation of the neurotransmission of L-DOPA may be elucidated now. L-DOPA is converted rapidly to DA in this system, elevating the cytosolic transmitter concentration. This in turn reduces the free energy required to provide neurotransmitter accumulation against the transmitter concentration gradient, resulting in a facilitation of vesicular transmitter uptake and an increased quantal size. It remains to be determined if this effect occurs in vivo, although it is consistent with results in brain slice preparations (Garris et al., 1994).

In summary, amperometric recordings from presynaptic sites demonstrate that quantal size in a central neuron can be modulated presynaptically by altering the number of molecules released per synaptic vesicle exocytic event. This would affect synapses adapted for transmitter overflow by increasing the spatial and temporal exposure of extrasynaptic sites to a given quantum. Both the advantages (high temporal resolution, usefulness for catecholamine detection, and direct measurement of quantal size) and drawbacks (low number of events recorded) of this technique follow from the detection of electroactive events only immediately under the electrode surface, because diff usional broadening would effectively filter the distal events. If the entire contents of a 50-nm-diameter synaptic vesicle were released during a single exocytic event, the intravesicular concentration in DA neurons exposed to GDNF or $20 \mu \mathrm{M}$ L-DOPA would be $\sim 300$ $\mathrm{mM}$; although this level is high, supraosmotic levels within vesicles may be achieved by precipitation with intravesicular anions (Rahamimoff and Fernandez, 1997).

\section{REFERENCES}

Almers W, Tse FW (1990) Transmitter release from synapses: does a preassembled fusion pore initiate exocytosis? Neuron 4:813-818.

Beck KD, Irwin I, Valverde J, Brennan TJ, Langston JW, Hefti F (1996) GDNF induces a dystonia-like state in neonatal rats and stimulates dopamine and serotonin synthesis. Neuron 16:665-673.

Bekkers JM, Richerson GB, Stevens CF (1990) Origin of variability in quantal size in cultured hippocampal neurons and hippocampal slices. Proc Natl Acad Sci USA 87:5359-5362.

Bruns D, Jahn R (1995) Real-time measurement of transmitter release from single synaptic vesicles. Nature 377:62-65.

Burke R, Antonelli M, Sulzer D (1998) Glial-derived neurotrophic factor prevents apoptosis of substantia nigra neurons during their period of natural cell death. J Neurochem, in press.

Chen G, Gavin PF, Luo G, Ewing AG (1995) Observation and quantitation of exocytosis from the cell body of a fully developed neuron in Planorbis corneus. J Neurosci 15:7747-7755.

Chow RH, von Ruden L (1995) Electrochemical detection of secretion from single cells. In: Single-channel recording (Sakmann B, Neher E, eds), pp 245-276. New York: Plenum.

Ciolkowski EL, Maness KM, Cahill PS, Wightman RM, Evans DH, Fosset B, Amatore C (1994) Disproportionation during electrooxidation of catecholamines at carbon-fiber microelectrodes. Anal Chem 66:3611-3617.

Durbec P, Marcos-Gutierrez CV, Kilkenny C, Grigoriou M, Wartiowaara K, Suvanto P, Smith D, Ponder B, Costantini F, Saarma M, Sariola H, Pachnis V (1996) GDNF signalling through the Ret receptor tyrosine kinase. Nature 381:789-793.

Edwards FA (1995) Anatomy and electrophysiology of fast central synapses lead to a structural model for long-term potentiation. Physiol Rev 75:759-787.

Finnegan JM, Pihel K, Cahill PS, Haung L, Zerby SE, Ewing AG,
Kennedy RT, Wightman RM (1996) Vesicular quantal size measured by amperometry at chromaffin, mast, pheochromocytoma, and pancreatic beta cells. J Neurochem 66:1914-1923.

Fon T, Pothos EN, Sun BC, Killeen N, Sulzer D, Edwards RH (1997) Vesicular transport regulates monoamine storage and release but is not essential for amphetamine action. Neuron 19:1271-1283.

Forti L, Bossi M, Bergamaschi A, Villa A, Malgaroli A (1997) Loosepatch recordings of single quanta at individual hippocampal synapses. Nature 388:874-878.

Frerking M, Borges S, Wilson M (1995) Variation in GABA mini amplitude is the consequence of variation in transmitter concentration. Neuron 15:885-895.

Garris PA, Ciolkowski EL, Pastore P, Wightman RM (1994) Efflux of dopamine from the synaptic cleft in the nucleus accumbens of the rat brain. J Neurosci 14:6084-6093.

Hebert MA, Van Horne CG, Hoffer BJ, Gerhardt GA (1996) Functional effects of GDNF in normal rat striatum: presynaptic studies using in vivo electrochemistry and microdialysis. J Pharmacol Exp Ther 279:1181-1190.

Jing S, Wen D, Yu Y, Holst PL, Luo Y, Fang M, Tamir R, Antonio L, Hu Z, Cupples R, Louis JC, Hu S, Altrock BW, Fox GM (1996) GDNFinduced activation of the Ret protein tyrosine kinase is mediated by GDNFR- $\alpha$, a novel receptor for GDNF. Cell 85:1113-1124.

Kang H, Schuman EM (1995) Long-lasting neurotrophin-induced enhancement of synaptic transmission in the adult hippocampus. Science 267:1658-1662.

Kullmann DM, Eredmil G, Asztely F (1996) LTP of AMPA and NMDA receptor-mediated signals: evidence for presynaptic expression and extrasynaptic glutamate spill-over. Neuron 17:461-474.

Kumer SC, Vrana KE (1996) Intricate regulation of tyrosine hydroxylase activity and gene expression. J Neurochem 67:443-462.

Leszczyszyn DJ, Jankowski JA, Viveros OH, Diliberto Jr EJ, Near JA, Wightman RM (1991) Secretion of catecholamines from individual adrenal medullary chromaffin cells. J Neurochem 56:1855-1863.

Lin L-FH, Doherty DH, Lile JD, Bektesh S, Collins F (1993) GDNF: a glial cell line-derived neurotrophic factor for midbrain dopaminergic neurons. Science 260:1130-1132.

Liu G, Tsien RW (1995) Properties of synaptic transmission at single hippocampal synaptic boutons. Nature 375:404-408.

Marshall DL, Redfern PH, Wonnacott S (1997) Presynaptic nicotinic modulation of dopamine release in the three ascending pathways studied by in vivo microdialysis: comparison of naive and chronic nicotinetreated rats. J Neurochem 68:1511-1519.

Mena MA, Davila V, Sulzer D (1997) Neurotrophic effects of L-DOPA in postnatal midbrain dopamine/cortical astrocyte co-culture. J Neurochem 69:1398-1408.

Mennerick S, Matthews G (1996) Ultrafast exocytosis elicited by calcium current in synaptic terminals of retinal bipolar neurons. Neuron 17:1241-1249.

Nirenberg MJ, Chan J, Liu Y, Edwards RH, Pickel VM (1997) Vesicular monoamine transporter-2: immunogold localization in striatal axons and terminals. Synapse 26:194-198.

Parsons TD, Lenzi D, Almers W, Roberts WM (1994) Calciumtriggered exocytosis and endocytosis in an isolated presynaptic cell: capacitance measurements in saccular hair cells. Neuron 13:875-883.

Petrenko AG (1993) $\alpha$-Latrotoxin receptor. Implications in nerve terminal function. FEBS Lett 325:81-85.

Pothos E, Desmond M, Sulzer D (1996) L-3,4-Dihydroxyphenylalanine increases the quantal size of exocytic dopamine release in vitro. J Neurochem 66:629-636.

Przedborski S, Khan U, Kostic V, Carlson E, Epstein CJ, Sulzer D (1996) Increased superoxide dismutase activity improves survival of cultured postnatal midbrain neurons. J Neurochem 67:1383-1392.

Rahamimoff R, Fernandez JM (1997) Pre- and postfusion regulation of transmitter release. Neuron 18:17-27.

Rayport S, Sulzer D, Shi W-X, Sawasdikosol S, Monaco J, Batson D, Rajendran G (1992) Identified postnatal mesolimbic dopamine neurons in cell culture: morphology and electrophysiology. J Neurosci 12:4264-4280.

Rebec GV, Pierce RC (1994) A vitamin as neuromodulator: ascorbate release into the extracellular fluid of the brain regulates dopaminergic and glutamatergic transmission. Prog Neurobiol 43:537-565.

Rieke F, Schwartz EA (1994) A cGMP-gated current can control exocytosis at cone synapses. Neuron 13:863-873. 
Ryan TA, Reuter H, Smith SJ (1997) Optical detection of a quantal presynaptic membrane turnover. Nature 388:478-482.

Schroeder TJ, Jankowski JA, Kawagoe KT, Wightman RM, Lefrou C, Amatore C (1992) Analysis of diffusional broadening of vesicular packets of catecholamines released from biological cells during exocytosis. Anal Chem 64:3077-3083.

Song HJ, Ming GL, Fon E, Bellocchio E, Edwards RH, Poo MM (1997) Expression of a putative vesicular acetylcholine transporter facilitates quantal transmitter packaging. Neuron 18:815-826.

Steyer JA, Horstmann H, Almers W (1997) Transport, docking, and exocytosis of single secretory granules in live chromaffin cells. Nature $474-478$

Stoop R, Poo MM (1996a) Synaptic modulation by neurotrophic factors. Prog Brain Res 109:359-364.

Stoop R, Poo MM (1996b) Synaptic modulation by neurotrophic factors: differential and synergistic effects of brain-derived neurotrophic factor and ciliary neurotrophic factor. J Neurosci 16:3256-3264.

Sulzer D, Rayport S (1990) Amphetamine and other psychostimulants reduce $\mathrm{pH}$ gradients in midbrain dopaminergic neurons and chromaffin granules: a mechanism of action. Neuron 5:797-808.

Sulzer D, Chen TK, Lau YY, Kristensen H, Rayport S, Ewing A (1995)
Amphetamine redistributes dopamine from synaptic vesicles to the cytosol and promotes reverse transport. J Neurosci 15:4102-4108.

Sulzer D, St. Remy C, Rayport S (1996) Reserpine inhibits amphetamine action in ventral midbrain culture. Mol Pharmacol 49:342-348.

Tang CM, Margulis M, Shi QY, Fielding A (1994) Saturation of postsynaptic glutamate receptors after quantal release of transmitter. Neuron 13:1385-1393.

Thoenen H (1995) Neurotrophins and neuronal plasticity. Science 270:593-598.

Tong G, Jahr CE (1994) Multivesicular release from excitatory synapses of cultured hippocampal neurons. Neuron 12:51-59.

Van der Kloot W (1991) The regulation of quantal size. Prog Neurobiol 36:93-130.

Van der Kloot W, Molgo J (1995) The relationship between quantal content and delayed quantal release. NeuroReport 6:1807-1810.

Wang T, Xie K, Lu B (1995) Neurotrophins promote maturation of developing neuromuscular synapses. J Neurosci 15:4796-4805.

Zhou Z, Misler S (1995) Amperometric detection of stimulus-induced quantal release of catecholamines from cultured superior cervical ganglion neurons. Proc Natl Acad Sci USA 92:6938-6942. 